\title{
Salvage use of venetoclax-based therapy for relapsed AML post allogeneic hematopoietic cell transplantation
}

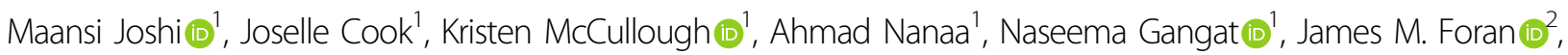 \\ Hemant S. Murthy ${ }^{2}$, Mohamed A. Kharfan-Dabaja $\mathbb{D}^{2}$, Lisa Sproat ${ }^{3}$, Jeanne Palmer ${ }^{3}$, Animesh Pardanani ${ }^{1}$, \\ Ayalew Tefferi', Kebede Begna', Michelle Elliot', Aref Al-Kali (10', Mrinal Patnaik $\mathbb{D}^{1}$, Mithun V. Shah (1)', \\ William J. Hogan (1), Mark R. Litzow ${ }^{1}$ and Hassan B. Alkhateeb (1)
}

\section{Dear Editor,}

Allogeneic hematopoietic cell transplantation (allo-HCT) is the only potentially curative treatment option available for patients with acute myeloid leukemia (AML) or myelodysplastic syndromes (MDS). Despite advances in optimizing conditioning regimens, graft-vs-host-disease (GVHD) management and supportive care, post-transplant relapse remains the Achilles heel impeding long-term survival ${ }^{1}$. Availability of effective therapies for these patients is an urgent area of unmet need.

BCL-2 is an anti-apoptotic protein and is present on leukocytes including leukemia stem cells. BCL-2 inhibitor venetoclax (VEN) has changed the treatment paradigm of AML in patients deemed unfit for intensive chemotherapy especially in frontline setting. Pollyea et al. $^{2}$ showed the synergistic effect of azacitidine and venetoclax in selectively inhibiting oxidative phosphorylation in leukemia stem cells which disrupts the energy metabolism resulting in cellular death. A phase III trial by DiNardo et al. ${ }^{3,4}$ recruited elderly newly diagnosed AML patients ( $\geq 65$ years) unfit for standard induction therapy. They showed a response rate of approximately $70 \%$ when treated with VEN in combination with a hypomethylating agent (HMA), a significant improvement on previously reported response rates of $20-30 \%$ with HMA alone $e^{5-7}$.

\footnotetext{
Correspondence: Hassan B. Alkhateeb (alkhateeb.hassan@mayo.edu) ${ }^{1}$ Division of Hematology, Department of Internal Medicine, Mayo Clinic, Rochester, MN, USA

${ }^{2}$ Division of Hematology \& Medical Oncology, Department of Medicine, Mayo Clinic, Jacksonville, FL, USA

Full list of author information is available at the end of the article

These authors contributed equally: Maansi Joshi, Joselle Cook
}

Although a prospective study is yet to be conducted, a moderate benefit has been suggested by retrospective studies in relapsed or refractory AML patients treated with VEN (or VEN-based regimens). A report of 43 patients treated with VEN in combination with HMA or low dose cytarabine (LDAC) demonstrated an overall response rate of $21 \%$ with 6-month overall survival of $24 \%{ }^{8}$. In another study of VEN + HMA in 33 patients with r/r AML, 13 of whom had previously undergone an allo- $\mathrm{HCT}$, the overall response rate was $64 \%$ with $50 \% \mathrm{CR}+\mathrm{CRi}$ and 1-year overall survival of $53 \%$. The response rate in patients who underwent a prior allo-HCT was $46 \%$ ( 6 out of 13 patients). The utility of VEN in the post-transplant setting remains poorly studied. Here, we report on our cohort of patients who underwent VEN-based therapy for post-allograft relapse.

After institutional review board approval, we conducted a retrospective analysis on consecutive patients with relapsed AML post allo-HCT who received VEN-based therapy in the salvage setting within the 3-site Mayo Clinic Cancer Center (Minnesota, Arizona, and Florida). Comprehensive disease and transplant history were recorded. Patients were risk stratified and response was reported according to the ELN 2017 guidelines ${ }^{10}$. Overall survival was defined from the date of initiation of venetoclax to date of death from any cause. Leukemia-free survival was calculated from date of initiation of venetoclax to date of disease progression or death. Statistical analysis and Kaplan-Meier curves were computed using JMP 14 software.

Between December 2017 and April 2020, 29 patients (52\% female) received VEN-based therapy for post-transplant relapse (Table 1). The median age at diagnosis was 58 years (range $20-72$ years). Eleven patients (38\%) had de novo 
Table 1 Baseline characteristics and response in patients salvaged with venetoclax.

\begin{tabular}{|c|c|c|c|c|}
\hline Baseline characteristic & Total $n=29$ & Responders & Non responders & $P$ value (Fisher's exact test) \\
\hline Age & 58 years ( $20-72$ years) & 58 years ( $20-64$ years) & 57 years $(25-72)$ & 0.08 \\
\hline Sex & & & & 0.2 \\
\hline Male & $14(48 \%)$ & $7(50 \%)$ & $7(50 \%)$ & \\
\hline Female & $15(52 \%)$ & $4(27 \%)$ & $11(73 \%)$ & \\
\hline AML type & & & & 0.8 \\
\hline De novo & $11(38 \%)$ & $5(45 \%)$ & $6(55 \%)$ & \\
\hline Secondary* & $5(17 \%)$ & $2(40 \%)$ & $3(60 \%)$ & \\
\hline $\mathrm{t}-\mathrm{AML}$ & $3(10 \%)$ & $1(33 \%)$ & $2(67 \%)$ & \\
\hline High-risk MDS & $10(35 \%)$ & $3(30 \%)$ & $7(70 \%)$ & \\
\hline Cytogenetic risk group & & & & 0.7 \\
\hline Favorable (inv 16) & $1(3 \%)$ & 0 & $1(100 \%)$ & \\
\hline Intermediate & $10(35 \%)$ & $4(36 \%)$ & 7 (64\%) & \\
\hline Adverse & $18(62 \%)$ & $7(39 \%)$ & $11(61 \%)$ & \\
\hline TP53 deletion or mutation & & & & 0.7 \\
\hline Yes & $12(41 \%)$ & $4(33 \%)$ & $8(67 \%)$ & \\
\hline No & $17(59 \%)$ & $7(41 \%)$ & $10(59 \%)$ & \\
\hline Median no. of therapies prior to transplant & 2 (range $1-4$ ) & 2 (range 1-4) & 2 (range $0-3$ ) & \\
\hline AML/MDS status at transplant & & & & 0.1 \\
\hline CR1 & $20(69 \%)$ & $8(47 \%)$ & $9(53 \%)$ & \\
\hline CR2 & $5(17 \%)$ & $3(60 \%)$ & $2(40 \%)$ & \\
\hline Persistent disease or MRD positive & $3(11 \%)$ & 0 & $3(100 \%)$ & \\
\hline Upfront transplant & $1(3 \%)$ & 0 & $1(100 \%)$ & \\
\hline Time to relapse post transplant & 9 months (range 2-37 months) & 15 (range 2-37 months) & 7.5 (range 2-35) & 0.4 \\
\hline Time to venetoclax initiation from transplant & 13 months (range $2-48$ ) & & & \\
\hline AML status at venetoclax initiation & & & & 0.8 \\
\hline Relapse 1 post transplant & $21(72 \%)$ & $8(38 \%)$ & $13(62 \%)$ & \\
\hline Relapse 2 post transplant & $4(14 \%)$ & $2(50 \%)$ & $2(50 \%)$ & \\
\hline Relapse 3 and/or refractory disease & $4(14 \%)$ & $1(25 \%)$ & $3(75 \%)$ & \\
\hline Median no. of venetoclax cycles & 2 (range 1-8) & & & \\
\hline Prior HMA exposure & & & & 0.4 \\
\hline Yes & $12(41 \%)$ & $3(25 \%)$ & $9(75 \%)$ & \\
\hline No & 17 (59\%) & $8(47 \%)$ & $9(53 \%)$ & \\
\hline
\end{tabular}

*AML secondary to underlying hematological disorder; $t-A M L$ therapy-related $A M L, C R$ complete remission, $M R D$ minimal residual disease by flow cytometry.

AML, 5 patients (17\%) had secondary AML post antecedent hematological disorder, 3 patients (10\%) had therapy-related disease, and 10 patients (35\%) had high-risk MDS. Twenty patients (69\%) had adverse risk cytogenetics, eight (28\%) had intermediate risk cytogenetics, and one had inversion 16. Molecular next-generation sequencing (NGS) data was available in 28 of 29 patients. TP53 mutation or deletion was seen in 12 patients (41\%) either at diagnosis or at relapse. In 7 patients, no pathogenic mutations were detected by NGS, they however had intermediate or adverse cytogenetic profile as per ELN classification. Other pathogenic mutations included epigenetic mutations (ASXL1, TET2, IDH1 \& 2, DNMT3A, SETBP1), RNA splicing mutations (SF3B1, SRSF2), transcription factor mutations (RUNX1, ETV6), activated signaling mutations (NRAS, JAK2), and tumor suppressor mutations (TP53, PHF6) (details in Supplementary Table 1). At transplant, 20 patients were in CR1, 5 in CR2, 3 had persistent disease, and one patient had upfront
allo-HCT for MDS. Fourteen patients received reduced intensity conditioning allo-HCT; the remaining 15 had myeloablative conditioning regimens. The donor source was matched unrelated donor $(n=17)$, matched related donor $(n=8)$, haploidentical $(n=2)$, and umbilical cord $(n=2)$. All patients received tacrolimus or cyclosporine plus methotrexate or mycophenolate-based GVHD prophylaxis, and four patients received ATG. Five patients had active GVHD at AML relapse, 2 with grade 1-2 GVHD were on steroids only, and 3 with grade 3-4 GVHD needed steroids with tacrolimus. The median follow-up was 16 months (range 2-50 months) post transplant.

Median time to relapse from transplant was 9 months (range 2-37 months). Twenty-one patients received VEN at first relapse post transplant, four were in second relapse, and four were in third relapse or had refractory disease. Five (17\%) of 29 patients had extramedullary relapse in addition to bone marrow relapse and two of those patients had CNS 
disease. Prior exposure to HMA and VEN was seen in 11 patients and 1 patient, respectively. Median number of VEN cycles was 2 (range 1-10). The median duration of cycle 1 was 27 days (range $1-67$ days; $n=29)$ ), median cycle 2 duration was 27.5 days (15-63 days; $n=12$ ), and of cycle 3 was 28 days (range $3-148$ days; $n=4$ ). All 29 patients required VEN dose adjustments due to concomitant antifungal prophylaxis with azoles due to inhibition of CYP3Amediated metabolism ${ }^{11}$. In 26 patients VEN was given with either decitabine $(n=18)$ for 5 days or azacitidine $(n=8)$ for 7 days at standard doses. One patient received VEN with concomitant low dose cytarabine, one patient received gilteritinib, and one patient had VEN monotherapy. The 2 patients with CNS disease had concomitant IT chemotherapy. Of the 3 patients able to complete 8 or more cycles, VEN dosing was shortened to 21 days of 28-day cycle (Pt 21), 14 days of a 28-day cycle (Pt 22), and one patient (Pt 25) had their decitabine changed to 3 days instead of 5 days to mitigate the hematologic toxicity. Cycle details are fully outlined in the Supplementary Tables 1 and 2. The proposed VEN dosing schedule was 28 days for all patients with MDS $(n=10)$; treatment details were evaluable for 9 patients. The median cycle 1 length was 32 days (range 8-67 days); four patients went on to cycle 2, median cycle length was 47.5 days (range 21-59), and two patients completed cycle 3 whose median length was 45 days (range 3-148).

The overall response rate was $38 \%(n=11)$ with eight patients (28\%) achieving complete remission (CR/CRi) and one a partial remission (PR) and two patients had a reduction in blast count. Median time to CR was 2.5 months (range 1-4 months) after a median of 2 cycles (range 1-3 cycles) to achieve best response. Median duration of response in those who responded was 7 months (range 1-11 months). Out of five patients with extramedullary disease, two (40\%) achieved CR, one with CNS disease and one with testicular and skin disease. Median overall survival after VEN initiation was 79 days (range 2-403 days) (Supplementary Table 3). Overall survival in responders vs nonresponders was 403 days (95\% CI 361-403) vs 55 days ( $95 \%$ CI 32-78) (log rank $p<0.0001$ ). Median leukemia free survival (LFS) in responders was 259 days (95\% CI 65-395) and in non-responders was 35 days (95\% CI 13-53) (log rank $p<0.0001$ ) (Fig. 1). Four of 12 patients (33\%) with TP53 mutation responded, 3 achieved CR and one patient had PR. One TP53 mutated patient in CR went on to have a second allograft and is currently in remission. There was, however, no difference in overall survival between those with TP53 mutation vs wild type $(p=0.8)$. At last follow up, 4 patients continue to be in CR, 2 still on VEN, one post second alloHCT, and one post high-dose cytarabine (HIDAC) and IT chemotherapy. Twenty-three patients progressed on VEN and received additional therapy or transitioned to hospice, and in 2 patients the response was unknown or not evaluable.

Grade 3 or 4 toxicities were neutropenia $(n=20)$, infections $(n=16)$, thrombocytopenia $(n=19)$ with diffuse alveolar hemorrhage seen in 2 patients, and anemia $(n=15)$. One patient had a confirmed invasive fungal infection (pulmonary aspergillosis) despite azole prophylaxis, ten patients had culture-positive bacterial infections, and 5 patients had culture-negative febrile neutropenia. Nineteen patients required cycle length adjustments due to hematological toxicity. This included shortening duration of VEN therapy to 14-21 days and/ or delaying subsequent cycles. There were no instances of GVHD flare while on VEN.

Treatment options for post-transplant relapse are often dictated by how 'robust' the patient is. Although clinical trials are always considered the preferred approach, often these patients are excluded, rendering their options limited to donor lymphocyte infusion (DLI), a second allo-HCT, or high-dose chemotherapy $(\mathrm{HDCT})^{12}$. Notably, DLI has
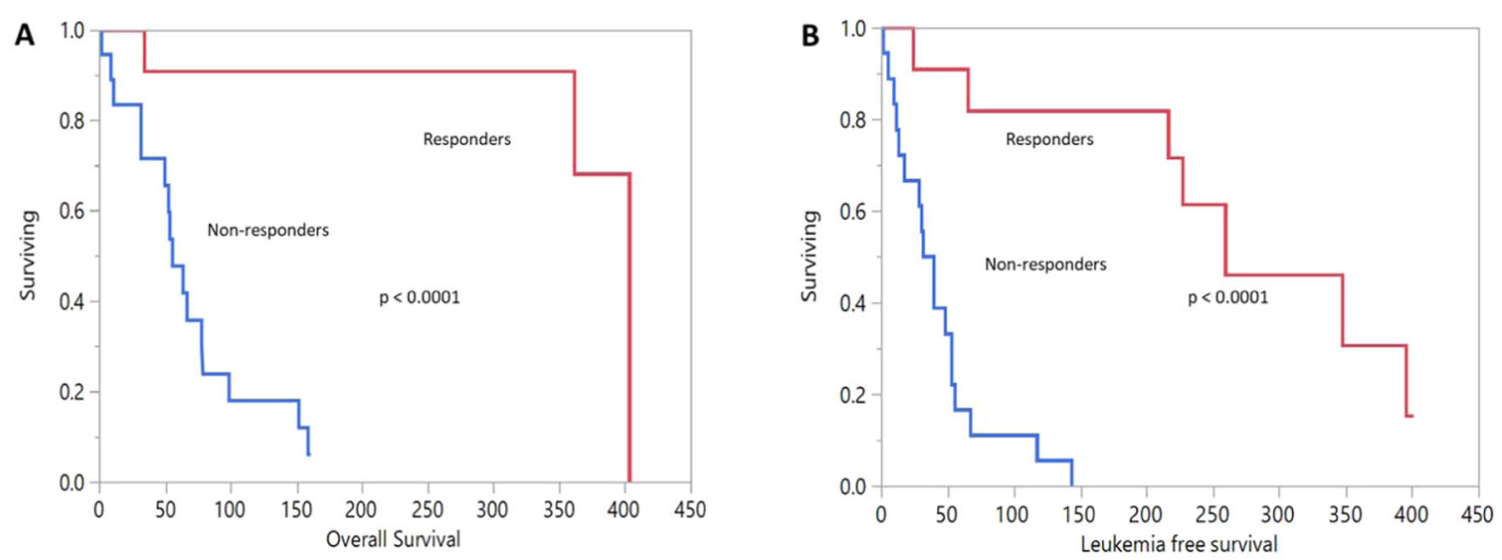

Fig. 1 Salvage use of venetoclax-based therapy for relapsed AML post allogeneic hematopoietic stem cell transplant. A Overall survival and B leukemia-free survival in responders vs non-responders in patients receiving VEN for post-transplant relapse. 
limited applicability in the setting of active GVHD, and patient's performance score is generally a limitation to pursuing a second allo-HCT owing to the resulting toxicities resulting from the allo-HCT. Chemotherapy, although associated with higher toxicity, results in higher response rates compared with HMA alone ${ }^{13}$. In our cohort VEN-based therapy yielded an ORR of 38\%, and prolonged both OS and LFS in responders. We noticed modest durable responses to this therapy with some activity in both TP53-mutated patients and those with extramedullary disease. Other groups have shown similar results with VEN use in high-risk patients ${ }^{14,15}$. A common challenge with VEN-based therapy is identifying the appropriate dose and cycle duration due to hematological toxicity. In our cohort, we noted most clinicians reduced duration of VEN and/or delayed subsequent treatment cycles when necessary. We were unable to determine the optimum dose to achieve best response while minimizing toxicity. Despite the small sample size and retrospective nature of our study, we provide evidence that even in the context of post-transplant relapse with adverse mutations or extramedullary disease, VEN-based therapy is capable of inducing $\mathrm{CR}$ and improving survival in responding patients. Future prospective studies focusing on appropriate patient selection and to define dose and duration of therapy are needed in this patient population.

\section{Author details}

'Division of Hematology, Department of Internal Medicine, Mayo Clinic, Rochester, MN, USA. ${ }^{2}$ Division of Hematology \& Medical Oncology, Department of Medicine, Mayo Clinic, Jacksonville, FL, USA. ${ }^{3}$ Division of Hematology, Department of Internal Medicine, Mayo Clinic, Scottsdale, AZ, USA

\section{Conflict of interest}

Dr. Al-Kali reports institutional research support by Celgene and Dr. Litzow reports research support by AbbVie. In addition, Dr. Kharfan-Dabaja discloses consultancy for Daiichi Sankyo Company and Pharmacyclics. No other authors report any conflicts of interests.

\section{Publisher's note}

Springer Nature remains neutral with regard to jurisdictional claims in published maps and institutional affiliations.

Supplementary information The online version contains supplementary material available at https://doi.org/10.1038/s41408-021-00437-z.
Received: 9 July 2020 Revised: 11 November 2020 Accepted: 14 January 2021

Published online: 04 March 2021

\section{References}

1. Bejanyan, N. et al. Survival of AML patients relapsing after allogeneic hematopoietic cell transplantation: a CIBMTR study. Biol. Blood Marrow Transpl. 21, 454-459 (2015)

2. Pollyea, D. A. et al. Venetoclax with azacitidine disrupts energy metabolism and targets leukemia stem cells in patients with acute myeloid leukemia. Nat. Med. 24, 1859-1866 (2018).

3. DiNardo, C. D. et al. Venetoclax combined with decitabine or azacitidine in treatment-naive, elderly patients with acute myeloid leukemia. Blood 133, 7-17 (2019).

4. DiNardo, C. et al. A randomized, double-blinded, placebo-controlled study of venetoclax with azacitidine vs azacitidine in treatment-naive patients with acute myeloid leukemia ineligible for intensive therapy-viale-A. EHA Haematol. LB2601, (2020).

5. Dombret, H. et al. International phase 3 study of azacitidine vs conventional care regimens in older patients with newly diagnosed AML with $>30 \%$ blasts. Blood 126, 291-299 (2015).

6. Roboz, G. J. et al. Results from a global randomized phase 3 study of Guadecitabine (G) vs treatment choice (TC) in 815 patients with treatment naïve (TN) AML unfit for intensive chemotherapy (IC) ASTRAL-1 study: analysis by number of cycles. Blood 134, 2591 (2019).

7. Konopleva, M. et al. A phase 2 study of ABT-199 (GDC-0199) in patients with acute myelogenous leukemia (AML). Blood 124, 118 (2014).

8. DiNardo, C. D. et al. Clinical experience with the BCL2-inhibitor venetoclax in combination therapy for relapsed and refractory acute myeloid leukemia and related myeloid malignancies. Am. J. Hematol. 93, 401-407 (2018).

9. Aldoss, I. et al. Efficacy of the combination of venetoclax and hypomethylating agents in relapsed/refractory acute myeloid leukemia. Haematologica 15, 404-407 (2018).

10. Döhner, H. et al. Diagnosis and management of AML in adults: 2017 ELN recommendations from an international expert panel. Blood 129, 424-448 (2017).

11. Agarwal, S. K. et al. Management of venetoclax-posaconazole interaction in acute myeloid leukemia patients: evaluation of dose adjustments. Clin. Ther. 39, 359-367 (2017).

12. Tsirigotis, P. et al. Relapse of AML after hematopoietic stem cell transplantation: methods of monitoring and preventive strategies. A review from the ALWP of the EBMT. Bone Marrow Transpl. 51, 1431-1438 (2016).

13. Motabi, I. H. et al. Chemotherapy versus hypomethylating agents for the treatment of relapsed acute myeloid leukemia and myelodysplastic syndrome after allogeneic stem cell transplant. Biol. Blood Marrow Transpl. 22, 1324-1329 (2016).

14. Aldoss, I. et al. Association of leukemia genetics with response to venetoclax and hypomethylating agents in relapsed/refractory acute myeloid leukemia. Am. J. Hematol. 24, 253-255 (2019).

15. Aldoss, I. et al. Venetoclax and hypomethylating agents in TP53 -mutated acute myeloid leukaemia. Br. J. Haematol. https://doi.org/10.1111/bjh.16166 (2019). 